\title{
Diagnosis of cervical plexus tumours by high-frequency ultrasonography
}

\author{
Wenqing Gong ${ }^{1+}$, Jing Wang ${ }^{1 \dagger}$, Liwei Huang ${ }^{2}, X$ Yang $^{3}$, Dingzhang Chen ${ }^{1 *}$ and Minjuan Zheng ${ }^{1 *}$
}

\begin{abstract}
Background: Cervical plexus (CP) tumours are difficult to diagnose because of atypical symptoms. This study aimed to summarize the features of a normal CP and CP tumours observed on high-frequency ultrasonography.

Methods: The ultrasound data of 11 CP tumour patients and 22 normal volunteers were collected. All 11 patients underwent magnetic resonance imaging (MRI), and 4 patients also underwent computed tomography (CT). The imaging data were compared with surgery and pathology data.

Results: The C7 vertebra and bifurcation of the carotid common artery (CCA) were useful anatomic markers for identifying the CP. In contrast to the C1 nerve (22.7\%), the C2-4 nerves were well displayed and thinner than the brachial plexus $(P<0.05)$. CP tumours were more common in females $(72.7 \%)$ and generally located at $C 4(72.7 \%)$ on the right side (81.8\%). Additionally, the nerve trunk in tumour patients was obviously wider than that in normal controls $(7.49 \pm 1.03 \mathrm{~mm}$ vs $2.67 \pm 0.36 \mathrm{~mm}, P<0.01)$. Compared with pathology, the diagnostic rates of CP tumours by MRI, CT and high-frequency ultrasound were $72.7 \%$ (8/11), 25\% (1/4) and 90.9\% (10/11), respectively.
\end{abstract}

Conclusions: The diagnosis of CP neuropathy is accurate and reliable by high-frequency ultrasound, and the C7 vertebra and bifurcation of the CCA are useful anatomic markers in CP ultrasonography.

Keywords: Cervical plexus, Tumour, High-frequency ultrasonography

\section{Background}

The cervical plexus $(\mathrm{CP})$ is located in the neck beneath the sternocleidomastoid muscle and comprises a coalition of nerves originating from $\mathrm{C} 1$ through $\mathrm{C} 4[1,2]$. Because of the higher location and atypical symptoms, most patients with $\mathrm{CP}$ tumor went to clinic for neck mass, some with concomitant pain, and $\mathrm{CP}$ masses are quite similar to lymph node or thyroid nodules upon palpation, so the presurgical diagnosis of these tumours is challenging [3]. The diagnosis of $\mathrm{CP}$ tumours relies on the clinical history, physical examination, electromyogram and diagnostic imaging [4]. Electromyogram was lack

\footnotetext{
*Correspondence: chendz2004@163.com; zhengmj@fmmu.edu.cn †Wenqing Gong and Jing Wang contributed equally to this work.

${ }^{1}$ Department of Ultrasound, Xijing Hospital, Fourth Military Medical University, No. 127 Changle West Road, Xi'an 710032, Shaanxi, China Full list of author information is available at the end of the article
}

of specific manifestation, magnetic resonance imaging (MRI) offers high-resolution visualization of CP tumours; however, the availability of MR neurography may be limited, and it is costly. Therefore, the clinical diagnosis of $\mathrm{CP}$ tumours remains difficult.

As a valuable technology, high-frequency ultrasonography provides superior imaging, with resolution up to $30 \mu \mathrm{m}$, which is sufficient for establishing the precise type and level of nerve injury [5]. We successfully used highfrequency ultrasound to diagnose brachial plexus (BP) closed injuries and neoplasms [6]. Previous studies on the ultrasonic imaging of a normal $\mathrm{CP}$ or the diagnosis of $\mathrm{CP}$ tumours are rare, with the exception of some case reports [7], and CP tumours are often misdiagnosed as lymph nodes $[3,8]$. Thus, this study aimed to summarize 


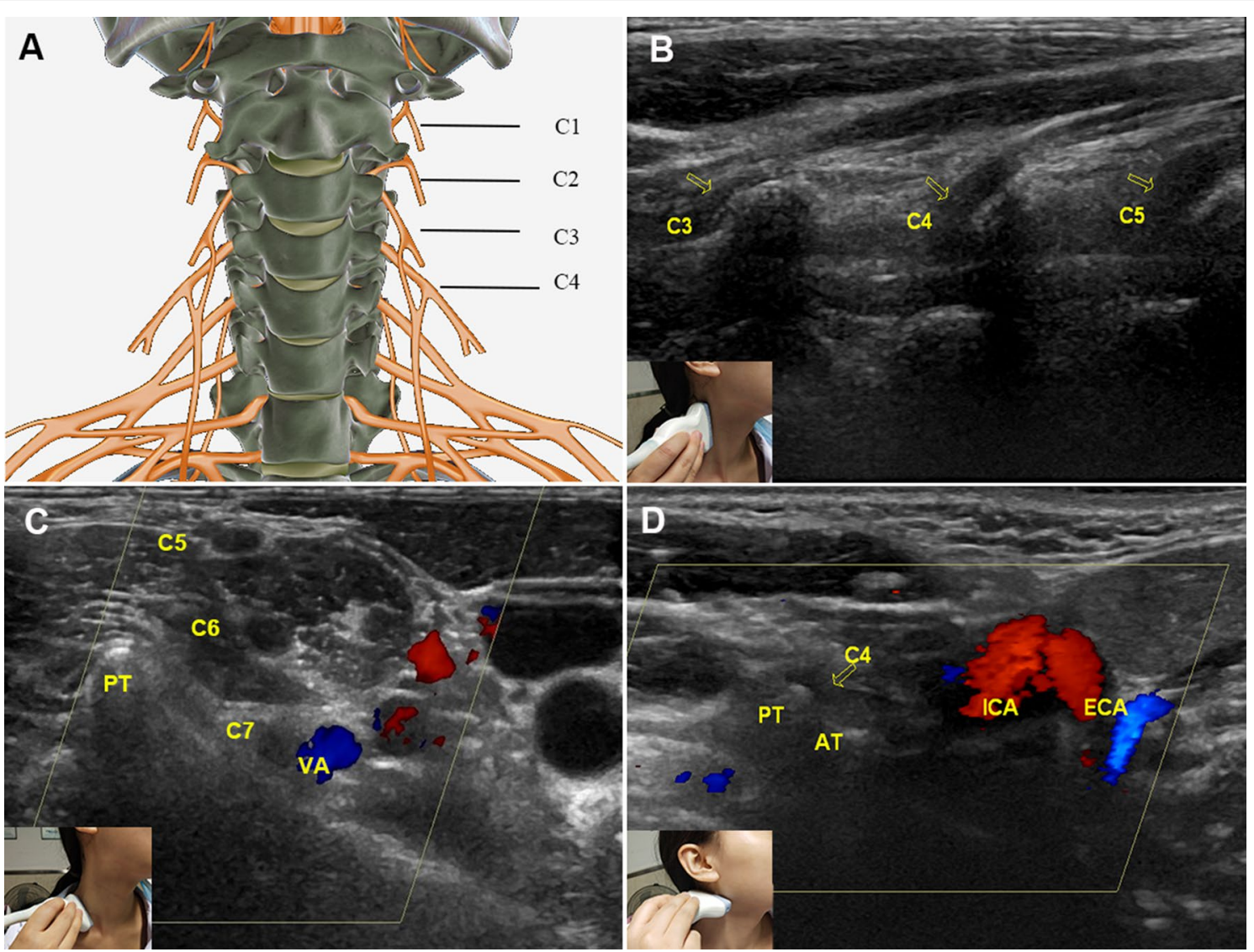

Fig. 1 Normal cervical plexus and its anatomical markers. A Anatomic diagram of the CP. B Longitudinal view of the C3-C5 roots (arrows), which appear as tubular hypoechoic structures with echogenic walls and a fibrillar texture. C The C7 vertebra was used as an anatomical marker to identify the CP. Arrows point to cervical nerve C7 (transverse view, arrow) and the PT. D The bifurcation of the common carotid artery served as another anatomical marker for identifying the CP (C4 level); the arrow indicates C4. PT posterior tubercle, AT anterior tubercle, VA vertebral artery, ECA external carotid artery, ICA internal carotid artery

the ultrasound characteristics of a normal $\mathrm{CP}$ and $\mathrm{CP}$ tumours to provide references for $\mathrm{CP}$ tumour diagnosis.

\section{Methods}

\section{Study subjects}

From January 2017 to December 2019, eleven patients with CP masses (8 females (72.7\%) and 3 males) who were diagnosed with $\mathrm{CP}$ tumours were enrolled. The patients were referred to the clinic for a palpable mass, arm numbness or pressing pain. All patients underwent high-resolution ultrasound neurography and MRI. Fourth patients also accepted Computed Tomography (CT) scanning. The average patient age was $40.73 \pm 13.24$ years $(15-54$ years). All 11 participants were confirmed to have $\mathrm{CP}$ tumours by surgery and pathology. Another 22 healthy adult volunteers (aged from 17 to $49,37.55 \pm 13.92$ years) were recruited to assess measurements of a normal cervical nerve.

\section{Equipment and methods}

MRI examinations were performed with the patient in the supine position with the head in a neutral position using a MAGNETOM Avanto 1.5 T MRI system (Siemens Healthcare, Erlangen, Germany). Sagittal T1WI, sagittal T2WI and axial T2WI for cervical spine were used as standard protocol. In addition, short inversion time inversion recovery sequences to suppress fat in paraspinal soft tissue, FLASH, and T1W FAT-SAT sequences were also included wherever necessary. 4 patients had taken a spiral CT scan (Somatom Definition Flash, Siemens Healthcare, Forchheim, Germany) from the top of the skull to the superior border of the aortic arch were reviewed. Images were reconstructed with a slice thickness of $1 \mathrm{~mm}$ and an increment of $0.8 \mathrm{~mm}$. All images were analyzed by experienced radiologist (Xu Yang, Liwei Huang). 
Table 1 Measurements of normal cervical nerve roots and nerves by ultrasonography ( $n=44,22$ subjects, bilateral)

\begin{tabular}{lccc}
\hline Cervical nerve & Trunk $(\mathbf{m m})$ & Root $(\mathbf{m m})$ & $\begin{array}{c}\text { Intervertebral } \\
\text { foramen level } \\
(\mathbf{m m})\end{array}$ \\
\hline Cervical plexus & & & \\
C2 & $2.46 \pm 0.31$ & $5.03 \pm 0.70$ & $7.65 \pm 1.36$ \\
C3 & $2.75 \pm 0.33$ & $5.86 \pm 0.82$ & $9.21 \pm 1.54$ \\
C4 & $2.83 \pm 0.39$ & $6.62 \pm 1.13$ & $11.18 \pm 7.44$ \\
Mean & $2.68 \pm 0.38^{*}$ & $5.84 \pm 1.04^{*}$ & $9.35 \pm 4.72^{*}$ \\
Brachial plexus & & & \\
C5 & $3.09 \pm 0.47$ & $7.33 \pm 1.22$ & $10.97 \pm 2.55$ \\
C6 & $3.48 \pm 0.39$ & $7.54 \pm 1.72$ & $11.21 \pm 2.90$ \\
C7 & $3.82 \pm 0.55$ & $7.57 \pm 1.09$ & $10.60 \pm 2.74$ \\
C8 & $3.79 \pm 0.61$ & $7.24 \pm 1.10$ & $10.40 \pm 2.58$ \\
Mean & $3.55 \pm 0.59$ & $7.42 \pm 1.31$ & $10.79 \pm 2.68$ \\
\hline
\end{tabular}

${ }^{*} P<0.05$, compared with the mean value of the brachial plexus

GE Logiq 9 ultrasound system with a 6-12 MHz linear array transducer (GE Medical Systems, Milwaukee, WI, USA). Ultrasonography was performed by two experienced sonographers (Jing Wang, Dingzhang Chen). The subjects were examined on both sides of the neck in the supine position. The CP nerve was fully scanned at the levels of the intervertebral foramen, roots, trunks, and visible terminal branches in the longitudinal and transverse planes. The diameters of the different levels of the CP nerve were measured in all subjects (healthy volunteers and those with $\mathrm{CP}$ tumours), and the size, echogenicity, Doppler blood flow and connection to the nerve of the masses were also evaluated.

Adler grading $[9,10]$ was used to classify Doppler blood flow in the tumour as follows: Adler 0 refers to that no obvious blood flow signal; Adler I refers to 1 or 2 small blood vessels with a diameter of $1 \mathrm{~mm}$ are detected; Adler II refers to that 3 or 4 small blood vessels are detected; Adler III refers to that more than 4 blood vessels, or the blood vessels are intertwined into a network are detected.

\section{Statistical analysis}

Statistical analyses were performed using statistical software (SPSS for Windows, version 21.0; SPSS, Chicago, IL, USA). Continuous variables are expressed as the mean \pm standard deviation (SD), and categorical variables are expressed as percentages. The variables were compared using the $t$ test or Fisher's exact test: $t$ test was used to compare diameter of normal CP and BP nerve, and Fisher's exact test was used to compare difference between mass location and colour Doppler grade. Probability $(P)$ values $<0.05$ were considered significant.
Table 2 Clinical and ultrasound features of cervical plexus tumours $(n=11)$

\begin{tabular}{ll}
\hline & n\%, mean \pm SD \\
\hline Mean age (years) & $40.73 \pm 13.24$ \\
Female & $72.7 \%(8)$ \\
Clinical manifestation & \\
Mass & $54.5 \%(6)$ \\
Arm numbness & $27.3 \%(3)$ \\
Pressing pain & $18.2 \%(2)$ \\
High-frequency ultrasound & \\
Right side & $81.8 \%(9)$ \\
Location of mass & \\
C4 & $72.7 \%(8)^{*}$ \\
C3 & $27.3 \%(3)$ \\
Diameter of mass (cm) & $4.83 \pm 1.62$ \\
Diameter of nerve trunk of the tumour (mm) & $7.49 \pm 1.03$ \\
Intervertebral foramen level (mm) & $12.70 \pm 1.90$ \\
Clear boundary with envelope integrity & $100 \%(11)$ \\
Colour Doppler grade & \\
I & $9.1 \%(1)^{*}$ \\
II & $54.5 \%(6)^{*}$ \\
II & $36.4 \%(4)$ \\
\hline
\end{tabular}

${ }^{*} P<0.05$, compared with grade and with the $\mathrm{C} 3$ location

\section{Results}

\section{Ultrasonography features of a normal CP nerve}

The ultrasound display rates of C2, C3 and C4 were 100\%, but that of $\mathrm{C} 1$ was $22.7 \%(5 / 22)$ due to its deep location (Fig. 1A, B). We found that two land markers were useful in identifying the CP. (1) The C7 vertebra: $\mathrm{C} 7$ is unique because it has a posterior tubercle only and can easily be found (Fig. 1C); then, C4-1 (above) can be confirmed from bottom to top. (2) Bifurcation of the common carotid artery (CCA): The bifurcation of the CCA is at the C4 level, which can be used as a land marker for $\mathrm{C} 4$ recognition (Fig. 1D). Normal CPs appeared on transverse views as round or elliptical hypoechoic structures with a hyperechoic rim. Viewed longitudinally, they appeared as tubular structures with a hypoechoic background (Fig. 1B). The diameter of the $\mathrm{CP}$ was less than that of the brachial plexus (Table 1).

\section{Ultrasonography features of $\mathrm{CP}$ tumours}

The clinical and ultrasound data of the 11 patients are shown in Table 2. The CP tumours presented as a single mass, and mainly located at $\mathrm{C} 4(72.7 \%, 8 / 11, P=0.037)$ on the right side $(81.8 \%, 9 / 11)$. Most tumours originated from the root level $(63.6 \%, 7 / 11)$, with a mean diameter of $4.83 \pm 1.62 \mathrm{~cm}$. The diameter of the connected root nerve was markedly increased compared with that of 

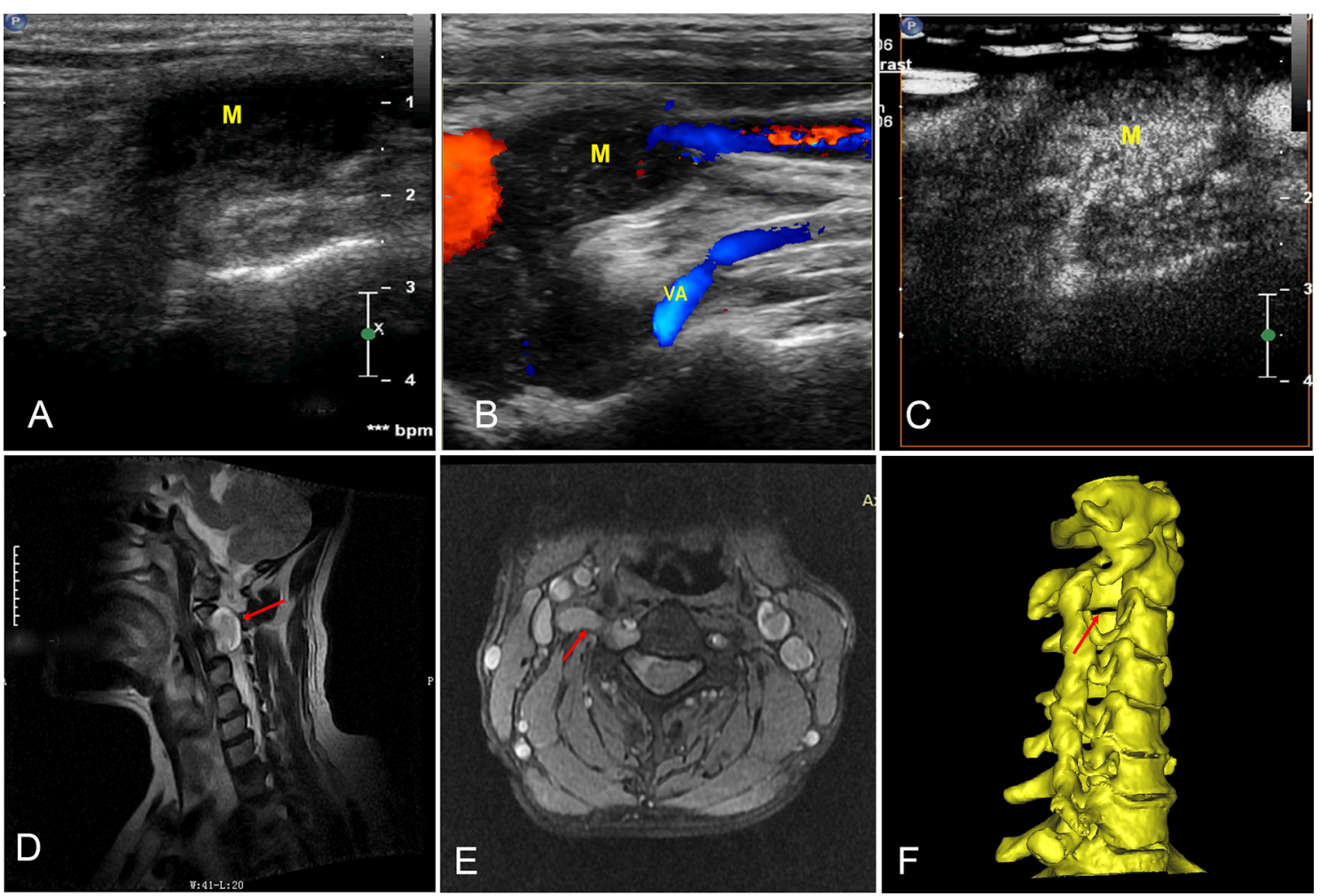

Fig. $2 \mathrm{MRI}, \mathrm{CT}$ and contrast ultrasound images of cervical plexus schwannomas (C3 level). A 2D ultrasonography of the transverse process of the vertebra, where $\mathrm{M}$ is the neuroma lesion originating from the intervertebral foramen. B Colour Doppler ultrasonography showing the blood flow signal in the mass. C Contrast-enhanced ultrasound showing rich blood perfusion. D, E MRI sagittal and cross-sections of a CP mass: the arrow indicates the lesion growing outwards from the intervertebral foramen. $\mathbf{F}$ The arrow indicates the enlarged intervertebral foramen (3D CT reconstruction)

the corresponding nerve on the healthy side because of oedema $(7.49 \pm 1.03 \mathrm{~mm}$ vs $2.67 \pm 0.36 \mathrm{~mm}, P<0.01)$. The intervertebral foramen was obviously enlarged.

In high-resolution ultrasound imaging, most tumours were homogeneously hypoechoic $(10,90.9 \%)$ with a clear boundary and envelope integrity (11, 100\%), and only one mass demonstrated an anechoic liquefaction zone. According to colour Doppler ultrasonography, moderate or strong blood signals were acquired from most tumours (grades II and III accounted for $90.9 \%$ of the total, and grade II alone accounted for $54.5 \%$ ). Two patients who underwent contrast-enhanced ultrasound showed abundant blood perfusion (Fig. 2A-C).

\section{$\mathrm{MRI}, \mathrm{CT}$ and pathology}

MRI (all patients) and CT (4 patients) showed CP masses with enlarged intervertebral foramens (Fig. 2D-F). Pathology confirmed that $72.7 \%$ (8/11) of tumours were schwannomas (Fig. 3), 2 were neurofibromas (18.2\%), and 1 was a spindle cell tumour (9.1\%). Compared with pathology, the diagnostic rates of CP tumours by MRI,
CT and high-frequency ultrasound were $72.7 \%(8 / 11)$, $25 \%(1 / 4)$ and $90.9 \%(10 / 11)$, respectively (Table 3$)$.

\section{Discussion}

Identification of the $\mathrm{CP}$ during ultrasound exam is challenging. According to our experience, two anatomical markers, the $\mathrm{C} 7$ vertebra and the bifurcation of the CCA, are very useful for $\mathrm{CP}$ identification. The $\mathrm{C} 7$ vertebra is unique because it has a posterior tubercle only, which easily distinguishes it from the other cervical vertebrae. The bifurcation of the CCA occurs at the C4 level. After C2$\mathrm{C} 4$ are identified ( $\mathrm{C} 1$ is usually difficult to identify), the probe can be transversely placed on the neck to show the structure of the cervical nerve root between the anterior and posterior tubercles, and then scanning is performed on the vertical section. Based on the overall scan, if a mass is connected to the thickened cervical nerves (caused by oedema), appearing as a "mouse tail sign" in the long-axis view, it can be considered to originate from the CP.

In our study, we first summarized the high-resolution ultrasound characteristics of $\mathrm{CP}$ tumours and found that 

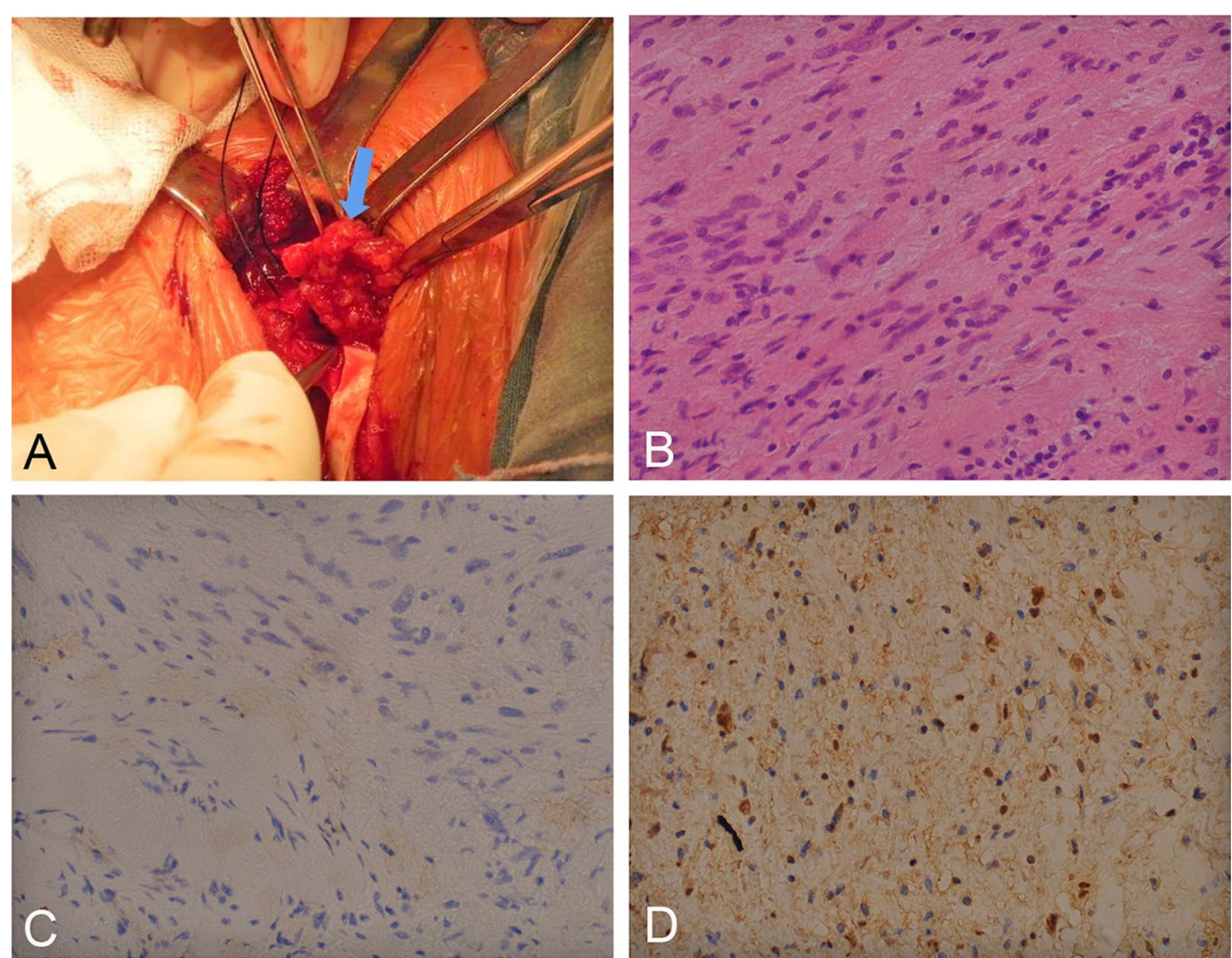

Fig. 3 Intraoperative view and pathology of schwannoma. A Intraoperative image shows the schwannoma in the C4 CP. The blue arrow indicates the neural lesion. B HE ( × 400) staining confirmed schwannoma. C, D NF and S-100 ( $\times 400)$ staining demonstrating nuclear and cytoplasmic immunoreactivity

$\mathrm{CP}$ tumours more commonly originate at $\mathrm{C} 4(72.7 \%)$ on the right side $(81.8 \%)$ and mainly in females $(72.7 \%)$. Most masses were homogeneously hypoechoic (90.9\%) with a clear boundary and envelope integrity (100\%). According to previous research, extracranial schwannomas present in the head and neck region account for $25-45 \%$ of all benign soft tissue tumours [11]. Malignant schwannomas are rare, accounting for only $5 \%$ of all soft tissue sarcomas [12]. The pathological results of our research confirmed that all the tumours were benign, with the proportion of schwannomas being $72.7 \%$. Colour Doppler ultrasonography showed that the blood flow signals of $90.9 \%$ of patients were grades II and III, demonstrating that benign $\mathrm{CP}$ tumours are abundant in blood vessels. Inside the tumours, rich perfusion was observed with contrast-enhanced ultrasound, indicating fast growth of these tumours.

The determination of whether tumours originate from the brachial plexus or $\mathrm{CP}$ or from other nerve branches in the neck, as well as the accurate location and diagnosis, is crucial for surgery $[13,14]$. Ultrasound plays an important role in the diagnosis of nerve masses. When patients experience cervical pain for unknown reasons, especially when a mass is found or when the limbs are numb, doctors should consider scanning both the $\mathrm{CP}$ and brachial plexus to differentiate between injuries, tumours, lymph node hyperplasia and metastasis. When masses are located in the paraspinal space, they are more likely to originate from the $\mathrm{CP}$ or brachial plexus rather than lymphoid tissues. Compared with the brachial plexus, the $\mathrm{CP}$ is shorter and thinner; therefore, $\mathrm{CT}$ and MRI are not satisfactory for demonstrating the $\mathrm{CP}$ as the origin of neuromas. For patients with cervical lumps or with pain upon palpation for a long time but without obvious lumps, the possibility of a high-position CP lesion should be considered. In this case, ultrasound can provide valuable information for the clinical strategy.

\section{Limitation}

There are limitations for this study. We included a small sample size of 11 subjects in this study. In addition, some patients did not undergo CT examination, and only 2 cases were done with contrast-enhanced ultrasound. In the future, we will continue to collect the imaging data of $\mathrm{CP}$ patients and look forward to providing reference for clinical diagnosis and surgical positioning. 
Table 3 CT, MRI and Pathology diagnosis of cervical plexus tumours

\begin{tabular}{|c|c|c|c|c|c|c|c|}
\hline Patients & Sex & Age (years) & $\begin{array}{l}\text { Mass location } \\
\text { (intraoperative) }\end{array}$ & Pathology & US $(n=11)$ & $C T(n=4)$ & $M R I(n=11)$ \\
\hline 1 & Male & 50 & C4 & Schwannoma & CP tumor & $\begin{array}{l}\text { Masses located between } \\
\text { the internal and external } \\
\text { carotid arteries }\end{array}$ & Neurogenic tumor (C4 level) \\
\hline 2 & Female & 15 & C3 & Neurofibromas & $\mathrm{CP}$ tumor & - & Neurogenic tumor (C3 level) \\
\hline 3 & Female & 24 & C4 & Spindle cell tumour & $\begin{array}{l}\text { Lymph nodes } \\
\text { enlargement? } \\
\text { CP tumor } \\
\text { can't be } \\
\text { excluded }\end{array}$ & - & Lymph nodes enlargement? \\
\hline 4 & Female & 51 & C3 & Schwannoma & CP tumor & - & Schwannoma (C3 level) \\
\hline 5 & Female & 28 & C4 & Schwannoma & CP tumor & - & $\begin{array}{l}\text { Neurogenic tumor (C3 or C4 } \\
\text { level) }\end{array}$ \\
\hline 6 & Female & 50 & C4 & Schwannoma & CP tumor & Carotid body tumor & Neurogenic tumor (C4 level) \\
\hline 7 & Female & 46 & $\mathrm{C} 4$ & Schwannoma & CP tumor & - & Neurogenic tumor (C4 level) \\
\hline 8 & Female & 54 & $\mathrm{C} 4$ & Schwannoma & CP tumor & $\begin{array}{l}\text { Mass behind the Sterno- } \\
\text { cleidomastoid muscle }\end{array}$ & Chemoreceptor Neoplasia \\
\hline 9 & Female & 45 & C4 & Neurofibromas & $\mathrm{CP}$ tumor & $\begin{array}{l}\text { Mass located in cervical } \\
\text { plexus (C3 or C4 level) }\end{array}$ & Neurogenic tumor (C4 level) \\
\hline 10 & Male & 51 & $\mathrm{C} 3$ & Schwannoma & $\mathrm{CP}$ tumor & - & Schwannoma (C3 or C4 level) \\
\hline 11 & Male & 34 & $\mathrm{C} 4$ & Schwannoma & CP tumor & - & $\begin{array}{l}\text { Lymph nodes enlargement? } \\
\text { Neurogenic tumor can't be } \\
\text { excluded }\end{array}$ \\
\hline \multicolumn{5}{|c|}{ Diagnosis sensitivity (\%) } & $90.90 \%$ & $25 \%$ & $72.70 \%$ \\
\hline
\end{tabular}

US ultrasound, $C T$ computed tomography, MRI magnetic resonance imaging

\section{Conclusion}

For $\mathrm{CP}$ nerve ultrasonography, the $\mathrm{C} 7$ vertebra and bifurcation of the CCA are useful anatomic markers in $\mathrm{CP}$ identify. CP tumours were more common in females and generally located at $\mathrm{C} 4$ on the right side. For well-trained sonographer, high-frequency ultrasound diagnosis sensitivity of $\mathrm{CP}$ neuropathy is accurate and reliable.

\section{Abbreviations}

CP: Cervical plexus; BP: Brachial plexus; MRI: Magnetic resonance imaging; CT: Computed tomography; CCA: Carotid common artery; SD: Standard deviation.

\section{Acknowledgements}

Thanks are due to Jing Wang for assistance with CTA/MRI data collection, ultrasound imaging of cervical plexus tumor acquire and paper writing, Liwe Huang and Xu Yang for imaging data collection, Minjuan Zheng and Dingzhang Chen for valuable discussion.

\section{Authors' contributions}

WG and JW carried out the studies, participated in collecting data, drafted the manuscript, and prepared figures $1-3, M Z$ and DC had revised the part of discussion. All authors have read and agreed to the published version of the manuscript.

\section{Funding}

This research was supported by the Xijing Innovation Fund (Grant Nos. XJGX15Y16 and XJGX15Y05).

\section{Availability of data and materials}

The datasets used and/or analyzed during the current study available from the corresponding author on reasonable request.

\section{Declarations}

Ethics approval and consent to participate

The design and protocol of this retrospective study were approved by the Ethics Committee of Xijing Hospital (KY20162034-1), and written informed consent was obtained from all participants. All methods in the study were carried out in accordance with the Helsinki guidelines and declaration.

\section{Consent for publication}

Written informed consent for publication was obtained from all participants.

\section{Competing interests}

All authors have no conflict of interest.

\section{Author details}

${ }^{1}$ Department of Ultrasound, Xijing Hospital, Fourth Military Medical University, No. 127 Changle West Road, Xi'an 710032, Shaanxi, China. ${ }^{2}$ Department of Special Clinic, Rehabilitation Center, Joint Logistics Support Force of PLA, Lintong 710600, Shaanxi, China. ${ }^{3}$ Department of Radiology, Xijing Hospital, Fourth Military Medical University, Xi'an 710032, Shaanxi, China.

Received: 1 July 2021 Accepted: 5 October 2021

Published online: 14 October 2021

\section{References}

1. Brown DL, Dellon AL. Surgical approach to injuries of the cervical plexus and its peripheral nerve branches. Plast Reconstr Surg. 2018;141(4):1021-5

2. Kohan EJ, Wirth GA. Anatomy of the neck. Clin Plast Surg. 2014;41(1):1-6.

3. Nagavalli S, Yehuda M, McPhaul LW, Gianoukakis AG. A cervical schwannoma masquerading as a thyroid nodule. Eur Thyroid J. 2017:6(4):216-20. 
4. Lee FC, Singh H, Nazarian LN, Ratliff JK. High-resolution ultrasonography in the diagnosis and intraoperative management of peripheral nerve lesions. J Neurosurg. 2011;114(1):206-11.

5. Forte AJ, Boczar D, Oliver JD, Sisti A, Clendenen SR. Ultra-high-frequency ultrasound to assess nerve fascicles in median nerve traumatic neuroma. Cureus. 2019;11(6):e4871.

6. Zheng MJ, Zhu YS, Zhou XD, Chen SS, Cong R, Chen DZ. Diagnosis of closed injury and neoplasm of the brachial plexus by ultrasonography. J Clin Ultrasound. 2014:42(7):417-22.

7. Gierek T, Zbrowska-Bielska D, Majzel K, Zygan L, Kajor M. Schwannoma of the cervical plexus: a case report. Otolaryngol Pol. 2003;57(4):569-72.

8. Ryu KH, Moon JI, Baek HJ, Cho SB, Choi BH, An HJ, Song DH. Brachial plexus schwannoma mimicking cervical lymphadenopathy: a case report with emphasis on imaging features. Medicine (Baltimore). 2018;97(42):e12880

9. Yang F, Zhao J, Liu C, Mao Y, Mu J, Wei X, Jia J, Zhang S, Xin X, Tan J. Superb microvascular imaging technique in depicting vascularity in focal liver lesions: more hypervascular supply patterns were depicted in hepatocellular carcinoma. Cancer Imaging. 2019;19(1):92.
10. Han H, Ding H, Ji Z, Zhang W, Wang Q, Wang W. Primary application of micro-flow imaging technology in the diagnosis of hepatic tumors. Ultrasound Med Biol. 2019;45(2):395-401.

11. Biswas D, Marnane CN, Mal R, Baldwin D. Extracranial head and neck schwannomas-a 10-year review. Auris Nasus Larynx. 2007;34(3):353-9.

12. Bailet JW, Abemayor E, Andrews JC, Rowland JP, Fu YS, Dawson DE. Malignant nerve sheath tumors of the head and neck: a combined experience from two university hospitals. Laryngoscope. 1991;101(10):1044-9.

13. Silver AJ, Mawad ME, Hilal SK, Ascherl GF Jr, Chynn KY, Baredes S. Computed tomography of the carotid space and related cervical spaces. Part II: neurogenic tumors. Radiology. 1984;150(3):729-35.

14. Chen D, Zheng M. Ultrasonography diagnosis of peripheral nerves: cases and illustrations. Singapore: Springer; 2020.

\section{Publisher's Note}

Springer Nature remains neutral with regard to jurisdictional claims in published maps and institutional affiliations.
Ready to submit your research? Choose BMC and benefit from:

- fast, convenient online submission

- thorough peer review by experienced researchers in your field

- rapid publication on acceptance

- support for research data, including large and complex data types

- gold Open Access which fosters wider collaboration and increased citations

- maximum visibility for your research: over $100 \mathrm{M}$ website views per year

At BMC, research is always in progress.

Learn more biomedcentral.com/submissions 